\title{
Alterations in juvenile diploid and triploid african catfish skin gelatin yield and amino acid composition: effects of chlorpyrifos and butachlor exposures
}

\begin{abstract}
Skin is a major by-product of the fisheries and aquaculture industries and is a valuable source of gelatin. This study examined the effect of triploidization on gelatin yield and proximate composition of the skin of African catfish (Clarias gariepinus). We further investigated the effects of two commonly used pesticides, chlorpyrifos (CPF) and butachlor (BUC), on the skin gelatin yield and amino acid composition in juvenile full-sibling diploid and triploid African catfish. In two separate experiments, diploid and triploid C. gariepinus were exposed for 21 days to graded CPF [mean measured: 10, 16, or $31 \mathrm{mg} / \mathrm{L}$ ] or BUC concentrations [Mean measured: 22, 44, or $60 \mathrm{mg} / \mathrm{L}$ ]. No differences in skin gelatin yield, amino acid or proximate compositions were observed between diploid and triploid control groups. None of the pesticide treatments affected the measured parameters in diploid fish. In triploids, however, gelatin yield was affected by CPF treatments while amino acid composition remained unchanged. Butachlor treatments did not alter any of the measured variables in triploid fish. To our knowledge, this study is the first to investigate changes in the skin gelatin yield and amino acid composition in any animal as a response to polyploidization and/or contaminant exposure.
\end{abstract}

Keyword: Clarias gariepinus; Pesticide; Polyploidy; Gelatin 